\title{
Staining pattern of type IV collagen and prognosis in early stage adenocarcinoma of the lung
}

\author{
N Watanabe, I Nakajima, S Abe, S Ogura, H Isobe, Y Kawakami
}

\begin{abstract}
Aims-To examine the prognostic value of basement membrane expression in early stage adenocarcinoma of the lung. Methods-Using antibodies to type IV collagen, basement membrane expression at the tumour-stromal border was immunohistochemically analysed in 30 patients with early stage adenocarcinoma of the lung (pstage I and pstage II). Two patterns of staining for type IV collagen were observed: in the first one the staining line was conserved or partially fragmented; in the second the staining line was widely fragmented or absent in more than $10 \%$ of the tumour area. The first staining pattern was categorised as continuous and the second as discontinuous. Results-Of the 24 patients with pstage I adenocarcinoma, $12(50 \%)$ cases showed a continuous pattern. In only one $(16 \cdot 7 \%)$ of the six patients with pstage II adenocarcinoma was this pattern evident. Five year survival was greater in pstage I adenocarcinoma (65\%) than in pstage II adenocarcinoma $(17 \%)$, but the difference was not significant. When the analysis was restricted to the 24 patients with pstage I adenocarcinoma, five year survival was better in continuous pattern cases $(88 \%)$ than in discontinuous pattern cases $(20.5 \%) \quad(p<0.05)$. The survival curve of 12 patients with pstage I adenocarcinoma and a discontinuous pattern resembled that of the six patients with pstage II adenocarcinoma.

Conclusion-These findings suggest that patients with pstage I adenocarcinoma and a discontinuous pattern have histopathologically unrecognised micrometastasis when they come to surgery. The staining pattern of type IV collagen could help in the prognosis of pstage I adenocarcinoma of the lung after surgery.
\end{abstract}

(F Clin Pathol 1994;47:613-615)

Medicine, Hokkaido

University, Japan

N Watanabe

I Nakajima

$S$ Abe

S Ogura

$\mathrm{H}$ Isobe

Y Kawakami

Correspondence to:

Naomi Watanabe, the Firs

Department of Medicine,

School of Medicine,

Hokkaido University, N15,

W7, Kitaku, Sapporo 060,

Japan

Accepted for publication

11 January 1994

The incidence of adenocarcinoma of the lung has gradually increased, ${ }^{12}$ but its pathological and biological behaviour is still not fully understood. At present, staging the disease based on the TNM system is the best predictor of prognosis. ${ }^{34}$ However, even if the disease is categorised as stage $I$, or even if the tumour is small and localised peripherally, tumour recurrence and metastasis are sometimes seen. But this could depend on tumour micrometastasis already being present when patients come to surgery.

The basement membrane is a ubiquitous extracellular matrix which separates the organ parenchymal cells from the interstitial stroma. The basement membrane constitutes the first natural barrier to the invasion of cancerous cells. During invasion or metastasis of cancer cells, the first step is penetration through the basement membrane, followed by migration into the interstitial stroma. ${ }^{5} \mathrm{~A}$ recent study of colorectal carcinomas has shown that extensive (continuous) expression of basement membrane is significantly related to a good prognosis. $^{6}$

In this study we investigated the possible correlation between the pattern of basement membrane expression and patient survival in early stage adenocarcinoma of the lung.

\section{Methods}

Tumour specimens from 30 patients (13 men and 17 women, average age 54.9 years) with early stage adenocarcinoma of the lung were analysed. They were obtained from surgically resected specimens at Hokkaido University Medical Hospital between 1970 and 1986. Surgical specimens were diagnosed histopathologically according to the World Health Organisation (WHO) classification. The histopathological findings and pTNM staging (pathological stage <pstage>) were grouped according to the International Union Against Cancer (UICC) classification. Twenty four cases were diagnosed as pstage I adenocarcinoma and six diagnosed as pstage II adenocarcinoma.

For histological and immunohistochemical evaluation, routinely formalin fixed and paraffin wax embedded tissue blocks were sectioned at $4 \mu \mathrm{m}$. Paraffin sections were waxed, rehydrated, and pretreated with pepsin $(0.4 \%$ in $0.01 \mathrm{M} \mathrm{HCl}$ for 120 minutes at room temperature) to restore immunoreactivity to type IV collagen. After blocking of the endogenous peroxidase with $3 \% \mathrm{H}_{2} \mathrm{O}_{2}$ and washing in phosphate buffered saline (PBS), the sections 

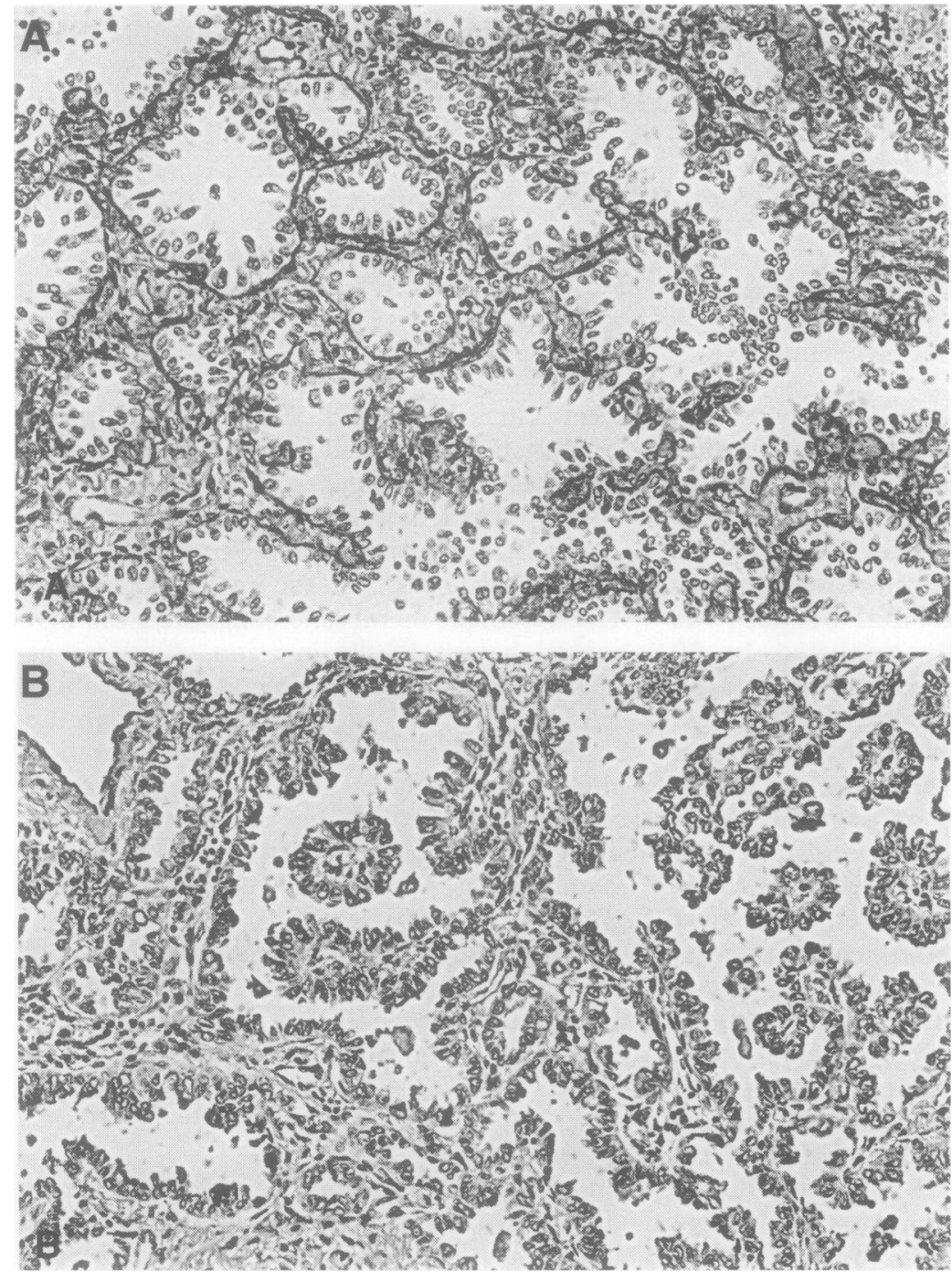

Figure 1 (A) Continuous staining pattern of type IV collagen in pstage I adenocarcinoma. (B) Discontinuous staining pattern of type IV collagen in pstage I adenocarcinoma.
The survival curves of the patients were drawn using the Kaplan-Meier method, and statistical evaluation was carried out using the Wilcoxon test.

\begin{abstract}
Results
All basement membranes in normal tissues were strongly and continuously stained using this method. But in the 30 lung adenocarcinomas different staining patterns were observed in the basement membrane, and the staining line looked either conserved or fragmented or even absent. As the staining pattern of type IV collagen was nearly always heterogeneous in a tumour we defined two main patterns. In the
\end{abstract}

Type IV collagen staining pattern in early stage adenocarcinoma

\begin{tabular}{lllll}
\hline Type IV collagen staining pattern & pstage & $\begin{array}{l}\text { No of } \\
\text { patients }\end{array}$ & Age(mean) & Differentiation* \\
\hline \multirow{2}{*}{ Continuous pattern } & I & 12 & $31-62(51 \cdot 9)$ & $6 / 3 / 3$ \\
& II & 1 & 77 & $0 / 1 / 0$ \\
Discontinuous pattern & I & 12 & $60-75(65 \cdot 0)$ & $2 / 8 / 2$ \\
& II & 5 & $54-64(58 \cdot 8)$ & $1 / 4 / 0$ \\
\hline
\end{tabular}

* Differentiation: well/moderately/poorly differentiated subgroup.

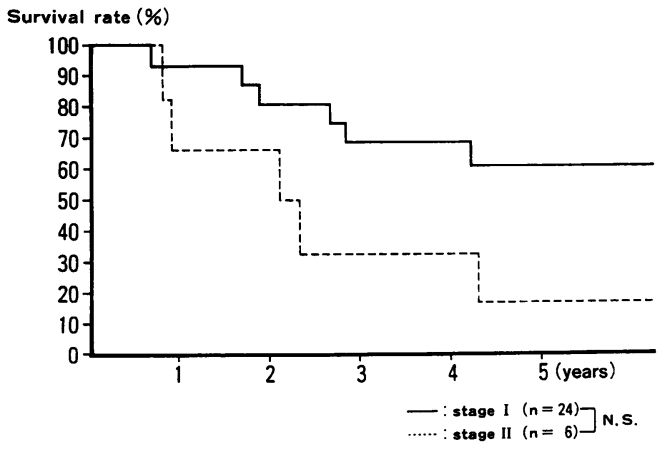

Figure 2 Survival curves of patients with pstage I and pstage II adenocarcinoma.

continuous pattern (fig 1A) staining was either conserved or occasionally fragmented in up to $10 \%$ of the tumour area. In the discontinuous pattern (fig 1B) staining was either absent or widely fragmented in more than $10 \%$ of the tumour area and, generally, the staining pattern was abnormal in over $80 \%$ of the tumour area. All our continuous pattern cases had over $90 \%$ normal staining (conserved or fragmented in a limited area of the tumour) and discontinuous pattern cases had over $80 \%$ abnormal staining (fragmented across extensive parts of the tumour foci, or absent).

Of the 24 patients with pstage I adenocarcinoma, $12(50 \%)$ showed a continuous pattern and $12(50 \%)$ a discontinuous pattern. Of the six patients with pstage II adenocarcinoma, only one $(16.7 \%)$ showed a continuous pattern (table).

Better five year survival trends were observed in the group with pstage I adenocarcinoma $(65 \%)$ than in the group with pstage II adenocarcinoma (17\%). However, no significant difference in survival was observed between the pstage I and II adenocarcinoma groups (fig 2).

When the analysis was restricted to the 24 patients with pstageI adenocarcinoma, a better five year survival was observed for patients with continuous patterns $(88 \%)$ than for patients with discontinuous patterns $(20.5 \%)$ $(p<0.05)$ (fig 3). The survival curve of 12 patients with pstage I adenocarcinoma and a discontinuous pattern of type IV collagen

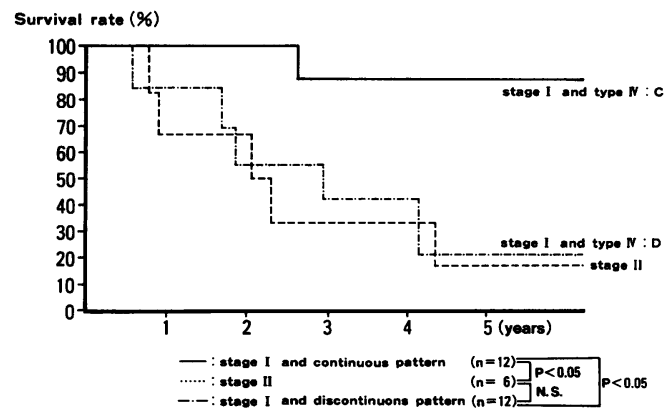

Figure 3 Survival curves of patients with pstage IC, pstage ID, and pstage II adenocarcinoma: pstage IC means pstage I and continuous pattern of type IV collagen; pstage ID means pstage I and discontinuous pattern of type IV collagen. 
staining resembled that of the six patients with pstage II adenocarcinoma (fig 3).

\section{Discussion}

Many authors have stated that TNM stage ${ }^{34}$ is an important prognostic factor in adenocarcinoma of the lung. The five year survival of patients with pstage I adenocarcinoma was only about $60-70 \% .^{78}$ However, tumour recurrence in patients whose disease was categorised as pstage I indicates the existence of prognostic factors other than TNM. Besides TNM-related factors, the following prognostic factors have been mentioned: histological subtype $^{9}$; degree of differentiation ${ }^{3410}$; vascular invasion ${ }^{310}$; the degree of collagenisation $^{311}$; mitotic index $;^{312}$ and nuclear DNA content. ${ }^{13}$

Recent reviews have shown that the changes in basement membrane distribution are related to prognosis in carcinomas of various organs, such as colorectal carcinoma ${ }^{6}$ and bladder carcinoma. ${ }^{14}$ But very few reports have been published about basement membrane expression in early stage lung cancer, ${ }^{15}$ especially stage I adenocarcinoma of the lung.

Invasion and metastasis are hallmarks of malignant neoplasia and frequently determine the course of the disease. Invasion precedes metastasis and interactions between tumour cells and the extracellular matrix are involved in this dynamic process. In carcinoma the first step is penetration through the epithelial basement membrane.

The difference of the type IV staining patterns observed in our cases was obvious and the intratumour heterogeneity of the staining was such that no mistakes could be made. The most interesting and important finding was the correlation of basement membrane staining pattern with prognosis in pstage I adenocarcinoma. The 12 patients with a continuous pattern of type IV staining had a significantly better prognosis than the 12 patients with a discontinuous pattern. Moreover, the survival curve of the 12 patients with a discontinous pattern in pstage I adenocarcinoma resembled that of the six patients with pstage II adenocrcinoma. These findings suggest that patients with pstage I adenocarcinoma and a discontinuous pattern have histopathologically unrecognised lymph node metastasis (micrometastasis) at the point of surgery.
From this limited study we cannot explain with certainty why prognosis in pstage I adenocarcinoma of the lung seems to be different between the two groups of patients, as defined by the staining pattern for type IV collagen.

However, several factors must be considered to understand the correlation between alterations in basement membrane components and poorer prognosis. In tumours there seems to be a balance between the synthesis of these components by epithelial malignant cells and the degradation of basement membrane by various tumour derived proteases. ${ }^{7}$ Extensive basement membrane expression in a neoplasm is probably a sign of a competent host response or of limited invasive potential, and might indicate low metastatic capability. ${ }^{6}$

We can't explain why this imbalance occurs in pstage I lung adenocarcinomas. We are now studying the expression of some tumour derived proteases to elucidate this.

1 Valaitis J, Warren S, Constable D, et al. Increasing incidence of adenocarcinoma of the lung. Cancer 1981 47:1042-6.

2 Vincent RG, Pickren JW, Lane WW, et al. The changing histopathology of lung cancer: a review of 1682 cases. Cancer 1977;39:1647-55.

3 Takise A, Kodama T, Shinomato Y, et al. Histopathologic prognosis factors in adenocarcinomas of the peripheral prognosis factors in adenocarcinomas of the peripheral

4 Lipford EI, Sears DL, Eggleston JC, et al. Prognostic factors in surgically resected limited stage, nonsmall cell carcinoma of the lung. Am F Surg Pathol 1984;8:357-65.

5 Liotta LA, Rao CN, Barsky SM, et al. Tumour invasion and extracellular matrix. Lab Invest 1983;49:636-49.

6 Havenith MG, Arends JW, Simon R, et al. Type IV collagen immunoreactivity in colorectal cancer. Prognostic value of basement membrane deposition. Cancer 1988;62. 2207-11.

7 Narke T, Goya T, Tanohiya R, et al. Prognosis and survival in resected lung carcinoma based on the new international staging system. If Thorac Cardiovasc Surg national stagin

8 Williams DE, Pairolero PC, Davis MS. Survival of patients surgically treated for stage I lung cancer. $\mathcal{f}$ Thorac Cardiovasc Surg 1981;82:70-6.

9 Campobasso O, Invernizzi, B, Musso M, et al. Survival rates of lung cancer according to histologic type. $\mathrm{Br} f$ Cancer 1974;29:240-6.

10 Chung CK, Zaino R, Stryker JA, et al. Carcinoma of the lung: evaluation of histological grade and factors influencing prognosis. Ann Thorac Surg 1981;33:599-604.

11 Shimosato Y, Hashimoto T, Kodema T, et al. Prognostic implication of fibrotic focus (scar) in small peripheral lung cancers. Am 7 Surg Pathol 1980;4:365-73.

12 James RD, Davey AT. Mitotic index in carcinomas of the lung. Lancet 1979;ii:852.

13 Isobe $\mathrm{H}$, Miyamoto $\mathrm{H}$, Shimizu $\mathrm{T}$, et al. Prognostic and therapeutic significance of the flow cytometric nuclear DNA content in non-small cell lung cancer. Cancer 1990;65:1391-5.

14 Daher N, Abourachid H, Bove N, et al. Collagen IV staining pattern in bladder carcinomas: relationship to prognosis. Br $\mathcal{F}$ Cancer 1987;55:665-71.

15 Grigioni WF, Biagini G, Garbias S, et al. Immunohistochemical study of basement membrane antigens in bronchioloalveolar carcinoma. Am F Pathol 1987;128: 217-24. 